\title{
On the association between type II radio bursts and CMEs
}

\author{
H. T. Claßen and H. Aurass
}

\author{
Astrophysikalisches Institut Potsdam, An der Sternwarte 16, 14482 Potsdam, Germany \\ e-mail: tclassen@aip.de
}

Received 23 July 2001 / Accepted 20 December 2001

\begin{abstract}
Ground-based observations of metric (coronal) type II radio bursts are compared with observations of coronal mass ejections (CMEs) obtained by the LASCO coronagraph aboard SOHO and with decametric (interplanetary) type II bursts recorded by the WAVES instrument aboard Wind. The basic data sample comprises 63 metric type II radio bursts observed during different phases of the solar cycle, i.e., 20 events in 1997 and 43 events in 1999/2000. Our analysis is based on temporal coincidence and velocity data derived from heighttime plots of CMEs and frequency drift rates of type II radio bursts, respectively. The results suggest that there are three scenarios leading to metric type II burst excitation: The radio emission is generated either at flarerelated blast wave shocks (class 1), at shocks driven by the leading edge of the CME (class 2), or at shocks driven by internal parts or the flanks of the CME (class 3). This classification is supported by an analysis of the association with $\mathrm{H} \alpha$ flare sites. The first class of events comprises 19 events (30\%), the second 19 events (30\%), and the third 18 events (29\%). 7 events (11\%) were not classified. Furthermore, we notice a possible solar cycle dependence of the relative occurrence of the different scenarios.
\end{abstract}

Key words. Sun: radio radiation - coronal mass ejections (CMEs) - shock waves

\section{Introduction}

Although it has been generally accepted that metric type II radio bursts are radio signatures of coronal MHDshock waves for more than 40 years (Uchida 1960; Wild 1962) the question of whether these shocks are flarerelated blast waves or shocks driven by CMEs as pistons is still under discussion (see e.g., Cliver et al. 1999). CMEs are regarded as observable changes in the coronal structure associated with new discrete white-light features. The time-scale of the structural change varies from minutes to hours. White-light observations of transients and CMEs have been performed by Skylab (e.g., Hildner 1977), SMM (e.g., Hundhausen et al. 1994), and most recently by SOHO/LASCO (St. Cyr et al. 2000). There are several investigations relating white-light observations of CMEs and radio observations (e.g., Sheeley et al. 1984; Bougeret 1985; Aurass 1997). The discussion of the significance of coronal type IIs was rekindled by carefully analysed ground-based radio observations (e.g., Mann et al. 1995, 1996) and by space-borne radio observations in the previously little-explored frequency range from $1-14 \mathrm{MHz}$, i.e., from decametric type IIs (e.g., Gopalswamy et al. 1998; Kaiser et al. 1998). According to these studies the situation is simple only for decametric type IIs. All of them are

Send offprint requests to: T. Claßen,

e-mail: tclassen@aip.de related to CMEs (Reiner et al. 2001b, 2001a) but in the reverse case only a few CMEs are accompanied by decametric type II observations, raising the question which kinds of CMEs are generating type II bursts. The spatial association between CMEs and the type II burst radio source has not yet been comprehensively studied (e.g., Gopalswamy 2000; Bastian et al. 2001). In the lower corona, the radio source can appear at any position behind or next to a possibly earlier or simultaneously launched CME body (e.g. Klein et al. 1997). For decametric type II bursts no radio imaging observations are available. The source is expected to be in front of the CME.

The radio emission process itself is due to plasma emission (Ginzburg \& Zheleznyakov 1958; Nelson \& Melrose 1985), i.e., a three-stage process starting with electrons accelerated at the moving shock. The electrons generate plasma oscillations which are subsequently transformed into radio waves. These radio waves are able to escape from the shock vicinity and can be observed in dynamic radio spectra as type II bursts (see Fig. 1). These bursts consist of one, two, or even more bands of enhanced radiation drifting slowly from high to low frequencies. The frequency of the plasma oscillations is given by the electron plasma frequency $f_{\mathrm{pl}}[\mathrm{Hz}]=8.98 \sqrt{n}_{\mathrm{e}}\left[\mathrm{m}^{-3}\right]$ with $n_{\mathrm{e}}$ as electron number density. The radio waves are observed at a multiple of this frequency since the plasma oscillations interact either with low frequency plasma waves generating 


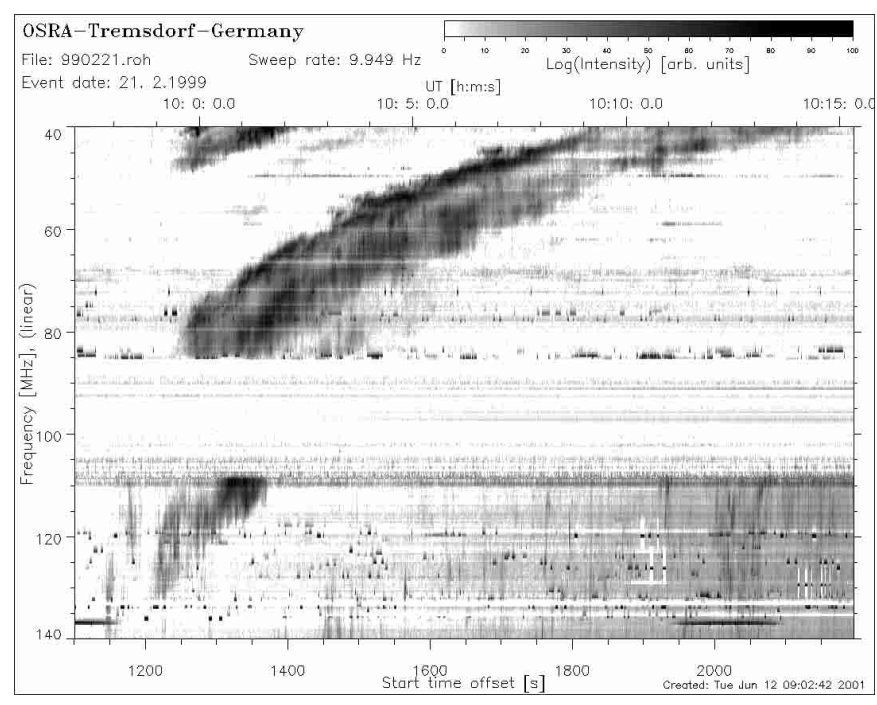

Fig. 1. Example of a dynamic radio spectrum (first event of Table 1 top). A pre-event spectrum is subtracted; the range $85-108 \mathrm{MHz}$ is disturbed by local transmitters. There are two pairs of fundamental/harmonic lanes starting at $50 />85 \mathrm{MHz}$ and 60 (very faint) $/ 140 \mathrm{MHz}$. In the same time interval a pair of CMEs is reported from the east and the west limb, respectively.

the fundamental emission, or with each other, generating the radio waves of the harmonic emission band. If the density distribution in the corona is known, i.e., $n_{\mathrm{e}}=n_{\mathrm{e}}(\boldsymbol{r})$, the observed frequency in a dynamic radio spectrum corresponds to a position in the solar corona and the frequency drift to the velocity of the exciter in the direction of the density gradient. In most cases one assumes a radial density behaviour, i.e., $n_{\mathrm{e}}(\boldsymbol{r})=n_{\mathrm{e}}(r)$ with empirical models. They are mostly based on white-light coronagraph observations (e.g., Newkirk 1961, 1967; Saito 1970, 1977).

Our analysis starts with a sample of 63 coronal type IIs observed with the radio spectrograph of the Astrophysikalisches Institut Potsdam. These data are combined with CME observations of the LASCO coronagraph aboard $\mathrm{SOHO}$ and decametric type IIs observed by the WAVES receiver aboard Wind. We took type IIs observed in different phases of the solar cycle, namely 20 events in 1997 (near solar minimum) and 43 events in 1999 and 2000 (near solar maximum). The first group has already been analysed with respect to EIT waves (Klassen et al. 2000). These authors found that $90 \%$ of the type II are associated with EIT waves. Although the type II drift rates yield a 2-3 times higher speed than the EIT waves both phenomena are signatures of the same fast magnetosonic disturbance (cf. Warmuth et al. 2001; Khan \& Aurass 2002).

The paper is organized in the following way. In the next section we briefly describe instrumentation and methods of data analysis. In particular we look at possible errors and uncertainties due to density models, projection effects, levels of analysis and so on. The results of the data analysis are presented in Sect. 3. This analysis leads to three different classes of metric type II events with respect to their association with CMEs. In Sect. 4 we interpret the results in relation to the different scenarios mentioned in the beginning of this section. We show that the different classes of type IIs are more or less closely related to solar surface phenomena, i.e., H $\alpha$ flares. The discussion includes also possible caveats with respect to visibility effects related to flare/CME localization. Furthermore we discuss a possible solar cycle dependence of the observed phenomena. In this regard the present study is intended to be a pilot study for a more detailed analysis with a bigger data set.

\section{Instruments, data set, and methods of analysis}

The starting point of our analysis are ground-based observations of coronal type IIs (http://www.aip.de./ People/AKlassen/) which are subsequently compared with LASCO (http://cdaw.gsfc.nasa.gov/CME_ist) and Wind/WAVES (http://lep694.gsfc.nasa.gov/ waves/waves.html) observations. The technical details of the instruments are well-known from previous publications. Thus the radio spectral polarimeter of the Astrophysikalisches Institut was introduced by Mann et al. (1992), the Large Angle Spectrometric Coronagraph (LASCO) aboard SOHO by Brueckner et al. (1995), and the radio receivers of Wind/WAVES by Bougeret et al. (1995). Since we are going to compare data from different radio receivers two things should be kept in mind. First, there is a frequency gap between 40 and $13.825 \mathrm{MHz}$, i.e., the Potsdam radio spectrograph consists of four sweep spectrographs in the ranges 40-90, 100-170, 200-400, and 400-800 MHz, while the receiver of WAVES lying next to this range (RAD2) covers $1.075-13.825 \mathrm{MHz}$. Secondly, the receivers have different sensitivities. On the one hand the Potsdam instrument measures relative fluxes and a lower limit of roughly 50 s.f.u. $=50 \times$ $10^{-22} \mathrm{Wm}^{-2} \mathrm{~Hz}^{-1}$ should be detectable at $40 \mathrm{MHz}$. On the other hand Dulk et al. (2000) and Reiner et al. (1998) report on absolute flux measurements with the WAVES receivers, and it seems that the results become significant at fluxes between $10^{3}$ and $10^{4}$ s.f.u. Thus it might not be surprising to find type IIs recorded by ground-based instruments that are no longer detected by Wind. This is very likely since we estimated the flux of most metric type IIs $(80 \%)$ to be of the order of $100-1000$ s.f.u.

Besides these hardware differences there are distinctions concerning the density models of the solar corona, as already mentioned in Sect. 1. The density models basically refer to mean values of the electron number density which have to be modified for coronal streamers and active regions by means of enhancement factors. For metric type IIs between roughly 90 and $40 \mathrm{MHz}$ the situation can be described as follows. A representative type II burst has a drift rate of $-0.1 \mathrm{MHz} / \mathrm{s}$ at the $70 \mathrm{MHz}$ level. This means for the different density models with corresponding enhancement factors a velocity/height difference of less than $10 \%$. The differences for the same model but with different enhancement factors are somewhat larger. They 
vary by a factor of 1.4 from the $1 \times$ to the $4 \times$ Newkirk model. Finally, it should be emphasized that we determine only the speed in the direction of the density gradient.

The analysis of the metric type IIs presented in this paper is based upon the $4 \times$ Newkirk (1961) model corresponding to the $10 \times$ Saito (1970) model. Although this enhancement factor seems to overestimate the shock velocity - some authors use a one- or twofold Newkirk model - a fourfold model seems to be reasonable (e.g., Cairns \& Robinson 1987; Robinson \& Stewart 1985). A physical justification might be the following. From a theoretical point of view it is most likely that type II emission is generated at shocks with a sufficiently high Mach number (see Sect. 4). That means that since the lower corona is highly structured by the solar magnetic field, the type II source region is tracing regions of low Alfvén speed and can deviate from a radial propagation. Thus, it is often observed that the source of a type II burst propagates obliquely through the solar corona (e.g., Klassen et al. 1999; Aurass et al. 1998). Assuming that the source travels at a $45^{\circ}$ angle in a $1 \times$ Newkirk region that is observed under the conditions of the previous paragraph we obtain a radial speed of $374 \mathrm{~km} \mathrm{~s}^{-1}$. The assumed geometry leads to a factor of $\sqrt{2}$ for the true shock velocity and we obtain a speed of $530 \mathrm{~km} \mathrm{~s}^{-1}$, close to the value for the $4 \times$ Newkirk model of $590 \mathrm{~km} \mathrm{~s}^{-1}$.

The analysis of LASCO and WAVES data faces the following constraints. Evaluating LASCO height-time plots one has to be aware of projection and of acceleration or deceleration effects. Decametric type II sources are likely traveling in a less structured magnetoplasma than the metric type II sources but they still need a density model for an interpretation of the spectra. In order to overcome the difficulties concerning projection effects of CMEs we need a localization of the associated CME source region with respect to the plane of the sky. These kinds of corrections are not included in our study and might be the reason for some uncertainty in the determination of the CME velocity (see Sect. 4). Furthermore there are hints of an acceleration/deceleration of CMEs but in most cases the linear fit of the height-time plots observed in the LASCO $\mathrm{C} 2-\mathrm{C} 3$ range is the best (St. Cyr, private communication). On the other hand one can see from the CME list at http://cdaw.gsfc.nasa.gov/CME_list that when acceleration/deceleration does occur it is typically of the order of $10-30 \mathrm{~m} / \mathrm{s}^{2}$. Taking this into account one can estimate that the CME velocity at typical metric type II heights $\left(1.5 R_{\odot}\right)$ might deviate from a linearly extrapolated CME velocity by roughly $100 \mathrm{~km} \mathrm{~s}^{-1}$. This uncertainty is in most cases smaller than the possible errors mentioned before. Thus we are basically going to discuss a situation as sketched in Fig. 2.

This figure illustrates the different levels of analysis. At first only the time difference between metric type II and white-light CMEs is taken into consideration. The time difference $\Delta t$ indicated in this figure is the difference between the time when the CME becomes visible in LASCO $\mathrm{C} 2$ and the time when the fundamental band

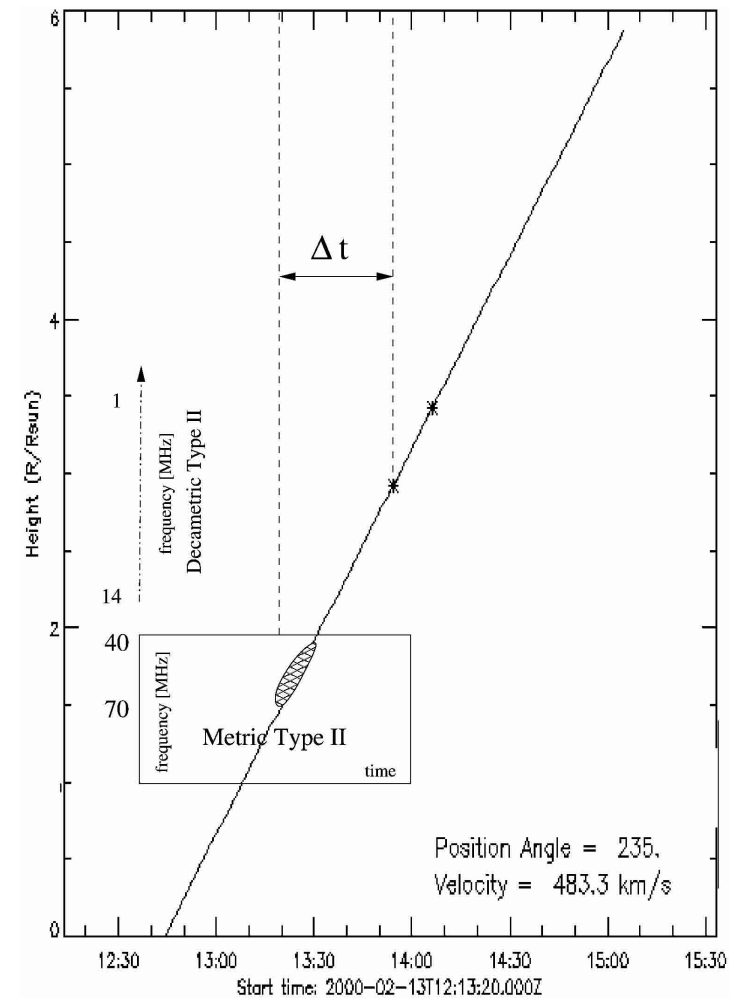

Fig. 2. A height-time plot of the February 13, 2000 CME. Stars on the regression line denote observed white light CME leading edge positions. Schematically, metric and decametric type II burst spectral ranges are inserted according to a fourfold Newkirk (1961) model.

of the type II burst is observed at $70 \mathrm{MHz}$ as reference level. The stars in Fig. 2 indicate the position of the CME leading edge. Next, we look at both height-time and velocity behaviour of metric type IIs and white-light CMEs. At this level of the analysis we are confronted with three different classes of type IIs. One group shows no obvious relation to CME observations and the other two classes seem to be directly CME related. Finally, the third step in our analysis takes both $\mathrm{H} \alpha$ flare observations and Wind/WAVES type II observations into account. The $\mathrm{H} \alpha$ flare site information was taken from the NOAA Solar Geophysical Data. In the case of a missing $\mathrm{H} \alpha$ report we checked Nançay radio heliograph data to determine the associated active region. This was done for 6 events ( 3 events from class 1, 3 events were not classified). We did not look for type II source sites; we checked only the position of the events reported by Nançay. Wind/WAVES data were obtained from the web http://lep694.gsfc.nasa.gov/waves/waves.html.

Presently we checked just the temporal coincidence, but some of the events have already been discussed in the literature (see references in Table 1).

Beside the choice of different phases of the solar cycle there was no bias in the type II selection. Therefore our sample also contains very faint bursts or bursts with rather complex spectral patterns. Thus further study 
involves different number of events for the different levels of analysis.

\section{Data analysis}

A simple way of relating coronal type IIs and white-light observations would be the comparison of the time difference between these phenomena as described in the previous section, i.e., the time difference $\Delta t$ between the first appearance of the $\mathrm{CME}$ in LASCO/C2 and the observation of the fundamental band of the metric type II at a reference level (here: $70 \mathrm{MHz}$, cf. Fig. 2). However, due to the LASCO observing schedule the uncertainty between the time of the true appearance and the first observation of the CME is about $20 \mathrm{~min}$. Therefore our analysis uses a linear extrapolation of the CME height-time plot (cf. Fig. 2) and determines the difference between the time when the type II burst was observed at the $70 \mathrm{MHz}$ plasma frequency corresponding to a heliocentric distance of $1.5 R_{\odot}$ and the time when the linearly extrapolated CME was at that height. The result of this analysis is shown in Fig. 3. There we display the time difference for the type IIs observed for periods of maximum (lower panel) and minimum (middle panel) solar activity, respectively. The upper panel displays the time difference for all analysed 63 type II events. The histograms are compressed for a time difference greater than two hours, i.e., we put all these events in the bins located around $\pm 120 \mathrm{~min}$. In total there are 12 events of this type, 7 in 1997 and 5 in $1999 / 2000$. Beside this artificial accumulation it is striking that there is a group of type IIs well associated with the CME observations. In 1999/2000 34 CMEs (out of 43) show a time difference between -60 and +60 min. This phenomenon is less pronounced for the 1997 type IIs where only 10 CMEs (out of 20) have been observed in this time interval. For the type IIs with the close temporal correlation the mean time difference is $\langle\Delta t\rangle=-8 \mathrm{~min}$ for the events in $1999 / 2000$ and $\langle\Delta t\rangle=-7$ min for the events in 1997.

Before discussing this situation in greater detail one must be aware of the following caveat. In principle each metric type II burst is associated with changes in the coronagraph's images. The detection of faint CMEs is easier at the limb of the solar disc (Cliver et al. 1999) and every CME is spatially and temporally structured - Hundhausen (1993) reports on a CME duration between a few minutes and several hours. At the present stage of the analysis however we are just analysing the kinematics of the leading edge of the emerging CMEs and we will discuss the problems concerning the type II localization in connection with CME visibility and $\mathrm{H} \alpha$ observations in Sect. 4.

Thus we took a somewhat closer look at those events presumably related to CMEs - those events with $|\Delta t|<$ $60 \mathrm{~min}$ - aiming at a detailed kinematical study of the movement of type II exciting shocks and CME leading edges. Starting with in total 44 events (34 in 1999/2000 and 10 in 1997) we are restricted to a sample of 37 events because we need type IIs showing both a fundamental and
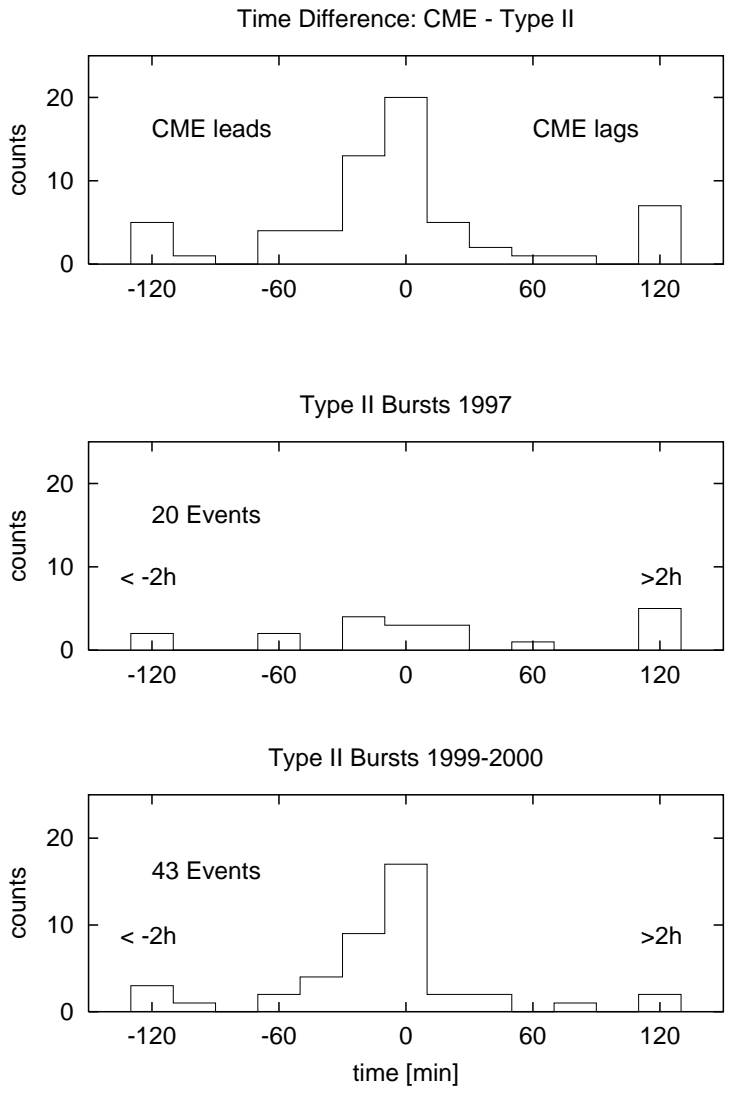

Fig. 3. Histogram of the time difference between the coronal type IIs at $70 \mathrm{MHz}$ plasma level and the extrapolated passage time of the $\mathrm{CME}$ at a heliocentric distance of $1.5 R_{\odot}$. The three panels show the behaviour during solar maximum (lower panel), solar minimum conditions (middle panel), and for all analysed events (upper panel).

harmonic band with a more or less well defined drift rate and clearly observed CMEs as well. These requirements reduce the original data set. The results are shown in Table 1 compiling the information for the coronal type IIs observed in 1999/2000 (28 events). The remaining type IIs observed in 1997 are available from the study of Klassen et al. (2000) and are not reproduced in this study. For completeness it should be mentioned that these 9 events were observed on April 7 (13:49), Sep. 28 (14:17), Oct. 7 (12:47); April 1 (13:49), May 25 (14:25), Oct. 9 (11:57), Nov. 3 (10:28), Nov. 6 (11:55), and Nov. 27 (13:16). The first three events belong to the upper part of Table 1; the last six events belong to the lower part.

The first column of Table 1 contains the date and time of the type IIs observed by the radio spectral polarimeter of the Astrophysikalisches Institut Potsdam. The time difference indicated in Col. 2 is obtained from the extrapolated CME appearance at the $70 \mathrm{MHz}$ plasma level and was used for Fig. 3. The time difference from Col. 3 is the difference between the first appearance of the type II radio burst and the maximum of the associated $\mathrm{H} \alpha$ flare. This information is used in Sect. 4 (see Fig. 4). Presently it should be realized that a negative time in Cols. 2 and 3 means that the CME leads the type II and that the 
Table 1. Summary of metric type II, CME, decametric type II, and H $\alpha$ observations in 1999/2000. Displayed are date and time of the metric type II radio bursts (Col. 1), and the time difference between these events and the temporally closest CMEs and $\mathrm{H} \alpha$ flare observations (Cols. 2 and 3). A negative $\Delta t$ indicates that the CME and metric type II are leading the associated event. Columns 4-6 contain the velocities of the associated metric type IIs, CMEs, decametric type IIs, and a comment on the individual transient - herringbones are fine structures of metric type II radio bursts and are regarded as radio signatures of electron beams accelerated at the type II generating shock. Column 7 shows the position and the importance of the H $\alpha$ flare, and Col. 8 shows where the CMEs have been observed and indicates the width of each CME. For further explanations see text.

Class 2 events 1999/2000

\begin{tabular}{|c|c|c|c|c|c|c|c|}
\hline $\begin{array}{l}\text { m-type II } \\
\text { Date/Time }\end{array}$ & $\begin{array}{c}\Delta t[\mathrm{~min}] \\
\mathrm{CME}-\mathrm{m} \text {-type II }\end{array}$ & $\begin{array}{c}\Delta t[\mathrm{~min}] \\
\mathrm{m} \text {-type II - } \mathrm{H} \alpha\end{array}$ & $\begin{array}{l}V_{\text {metr. }}\left[\mathrm{km} \mathrm{s}^{-1}\right] \\
\text { comment }\end{array}$ & $\begin{array}{l}V_{\mathrm{CME}}\left[\mathrm{km} \mathrm{s}^{-1}\right] \\
\text { comment }\end{array}$ & $\begin{array}{l}V_{\text {Dmetr. }}\left[\mathrm{km} \mathrm{s}^{-1}\right] \\
\text { comment }\end{array}$ & $\begin{array}{l}\text { H } \alpha \text {-Pos. } \\
\text { Imp. }\end{array}$ & $\begin{array}{l}\text { CME-Pos. } \\
\text { Width [deg] }\end{array}$ \\
\hline $\begin{array}{l}\text { 21 Feb. } 1999 \\
09: 57-10: 14\end{array}$ & $\overline{-1-8 .}$ & $\bar{~} 59.8$ & $\begin{array}{c}500 \pm 60 \\
4 \text { main bands }\end{array}$ & $\begin{array}{c}461 \\
2 \text { simultaneous eject. }\end{array}$ & $\begin{array}{l}- \\
\text { no obs. }\end{array}$ & $\begin{array}{l}\text { N19W45 } \\
\text { SF }\end{array}$ & $\begin{array}{c}E / W \\
16\end{array}$ \\
\hline $\begin{array}{l}15 \text { Mar. } 1999 \\
06: 45-06: 55 \\
\end{array}$ & 2. & -19.7 & $\begin{array}{c}550 \pm 150 \\
\text { complex }\end{array}$ & $\begin{array}{c}624 \pm 40 \\
\text { highly structured } \\
\end{array}$ & $\begin{array}{c}- \\
\text { no obs. }\end{array}$ & $\begin{array}{c}\text { N17E19 } \\
\text { SF }\end{array}$ & $\overline{90}$ \\
\hline $\begin{array}{l}27 \text { May } 1999 \\
10: 48-10: 54\end{array}$ & -16 & -19 & $\begin{array}{l}1,280 \pm 300 \\
\text { diffuse }\end{array}$ & $\begin{array}{c}1,149 \\
\text { backside event }\end{array}$ & $\begin{array}{c}- \\
10: 55\end{array}$ & $\begin{array}{l}\text { N31W06 } \\
\text { SF }\end{array}$ & $\begin{array}{l}\text { halo? } \\
360\end{array}$ \\
\hline $\begin{array}{l}\text { 31 May } 1999 \\
09: 40-09: 48\end{array}$ & -1 & 3.3 & $\begin{array}{c}550 \pm 60 \\
\text { starts at } 60 \mathrm{MHz}\end{array}$ & $\begin{array}{c}435 \pm 90 \\
\text { Cloud of material }\end{array}$ & $\begin{array}{c}- \\
\text { no obs. }\end{array}$ & $\begin{array}{c}\text { N18W27 } \\
\text { SF }\end{array}$ & $\begin{array}{c}\text { halo? } \\
360\end{array}$ \\
\hline $\begin{array}{l}29 \text { Jun. } 1999 \\
05: 15-05: 22\end{array}$ & -28 & -6.0 & $\begin{array}{c}580 \\
\text { faint, diffuse }\end{array}$ & $\begin{array}{c}589 \\
\text { diffuse front }\end{array}$ & no obs. & $\begin{array}{c}\text { N18E07 } \\
\text { SF }\end{array}$ & $\begin{array}{l}\text { halo } \\
360\end{array}$ \\
\hline $\begin{array}{l}30 \text { Jun. } 1999 \\
11: 30-11: 35\end{array}$ & -6 & -2.4 & $\begin{array}{c}840 \\
\text { mainly herringbones }\end{array}$ & $\begin{array}{c}627 \\
\text { faint fuzzy }\end{array}$ & $\begin{array}{c}- \\
\text { no obs. }\end{array}$ & $\begin{array}{l}\text { S15E00 } \\
1 \mathrm{~B}\end{array}$ & $\begin{array}{l}\text { halo } \\
360\end{array}$ \\
\hline $\begin{array}{l}16 \text { Jul. } 1999 \\
15: 54-16: 08\end{array}$ & -18 & - & $\begin{array}{c}850 \\
\text { multiple lanes }\end{array}$ & $\begin{array}{c}46 \\
\text { large bright loop }\end{array}$ & $\begin{array}{c}- \\
\text { no obs. }\end{array}$ & $\begin{array}{l}- \\
-\end{array}$ & $\begin{array}{l}\text { NW } \\
111\end{array}$ \\
\hline $\begin{array}{l}08 \text { Sep. } 1999 \\
12: 18-12: 21\end{array}$ & -6 . & - & $\begin{array}{c}520 \\
\text { mainly herringbones }\end{array}$ & $\begin{array}{c}412 \\
\text { fuzzy loop }\end{array}$ & no obs. & $\begin{array}{l}- \\
-\end{array}$ & $\begin{array}{c}E \\
98\end{array}$ \\
\hline $\begin{array}{l}20 \text { Oct. } 1999 \\
09: 30-09: 40\end{array}$ & 2. & 3.0 & $\begin{array}{c}450 \\
\text { faint, fades at } 70 \mathrm{MHz}\end{array}$ & $\begin{array}{c}486 \\
\text { faint fuzzy }\end{array}$ & $\begin{array}{c}- \\
\text { no obs. }\end{array}$ & $\begin{array}{c}\text { S14E78 } \\
\text { SF }\end{array}$ & $\begin{array}{l}\text { SE } \\
51\end{array}$ \\
\hline $\begin{array}{l}27 \text { Oct. } 1999 \\
13: 25-13: 38\end{array}$ & -9. & 0.6 & $\begin{array}{c}500 \\
\text { faint, multiple lanes }\end{array}$ & $\begin{array}{c}411 \\
\text { loop with core }\end{array}$ & no obs. & $\begin{array}{l}\text { S16W85 } \\
\text { SF }\end{array}$ & $\begin{array}{c}\mathrm{E} \\
94\end{array}$ \\
\hline $\begin{array}{l}29 \text { Dec. } 1999 \\
09: 35-09: 39\end{array}$ & -25 & 9.3 & $\begin{array}{c}345 \\
\text { intermittent }\end{array}$ & $\begin{array}{c}404 \\
\text { large loop }\end{array}$ & $\begin{array}{c}- \\
\text { no obs. }\end{array}$ & $\begin{array}{l}\text { N24W66 } \\
1 \mathrm{~N}\end{array}$ & $\begin{array}{l}\text { NW } \\
70\end{array}$ \\
\hline $\begin{array}{l}08 \text { Feb. } 2000 \\
08: 53-09: 16\end{array}$ & -8 & -3.5 & $\begin{array}{c}630 \pm 70 \\
\text { complex, multiple lanes }\end{array}$ & $\begin{array}{c}715 \\
\text { bright struct. loop }\end{array}$ & $\begin{array}{c}620 \\
\text { Reiner et al. (2001b) }\end{array}$ & $\begin{array}{l}\text { N25E26 } \\
1 \mathrm{~N}\end{array}$ & $\begin{array}{l}\text { halo } \\
360\end{array}$ \\
\hline $\begin{array}{l}18 \text { Feb. } 2000 \\
09: 19-09: 49\end{array}$ & 1. & -18.5 & $\begin{array}{c}860 \pm 100 \\
\text { starts at } 60 \mathrm{MHz}\end{array}$ & $\begin{array}{c}914 \\
\text { large bright loop }\end{array}$ & no obs. & $\begin{array}{l}\text { S16W78 } \\
\text { SF }\end{array}$ & $\begin{array}{c}\mathrm{W} \\
118\end{array}$ \\
\hline $\begin{array}{l}19 \text { Feb. } 2000 \\
08: 40-08: 57\end{array}$ & 2. & -19.5 & $\begin{array}{c}595 \\
\text { faint, intermittent }\end{array}$ & $\begin{array}{c}366 \\
\text { Material eject. }\end{array}$ & $\begin{array}{c}- \\
\text { no obs. }\end{array}$ & $\begin{array}{c}\text { S22W38 } \\
\text { SF }\end{array}$ & $\begin{array}{c}\text { SW } \\
52\end{array}$ \\
\hline $\begin{array}{l}19 \text { Mar. } 2000 \\
11: 44-11: 45\end{array}$ & 12. & -12.0 & $\begin{array}{c}360 \\
\text { no obs. } 40-90 \mathrm{MHz}\end{array}$ & $\begin{array}{c}347 \\
\text { rough lead. edge }\end{array}$ & no obs. & $\begin{array}{l}\text { S20W69 } \\
\text { SF }\end{array}$ & $\begin{array}{l}\text { SW } \\
21\end{array}$ \\
\hline $\begin{array}{l}04 \text { Apr. } 2000 \\
15: 29-15: 31\end{array}$ & -4 & -8.3 & $\begin{array}{c}900 \pm 300 \\
\text { mainly herringbones }\end{array}$ & $\begin{array}{c}1,136 \pm 200 \\
\text { bright front }\end{array}$ & $\begin{array}{c}- \\
15: 45\end{array}$ & $\begin{array}{l}\text { N15W63 } \\
2 \mathrm{~F}\end{array}$ & $\begin{array}{l}\text { halo } \\
360\end{array}$ \\
\hline
\end{tabular}

Class 3 events 1999/2000

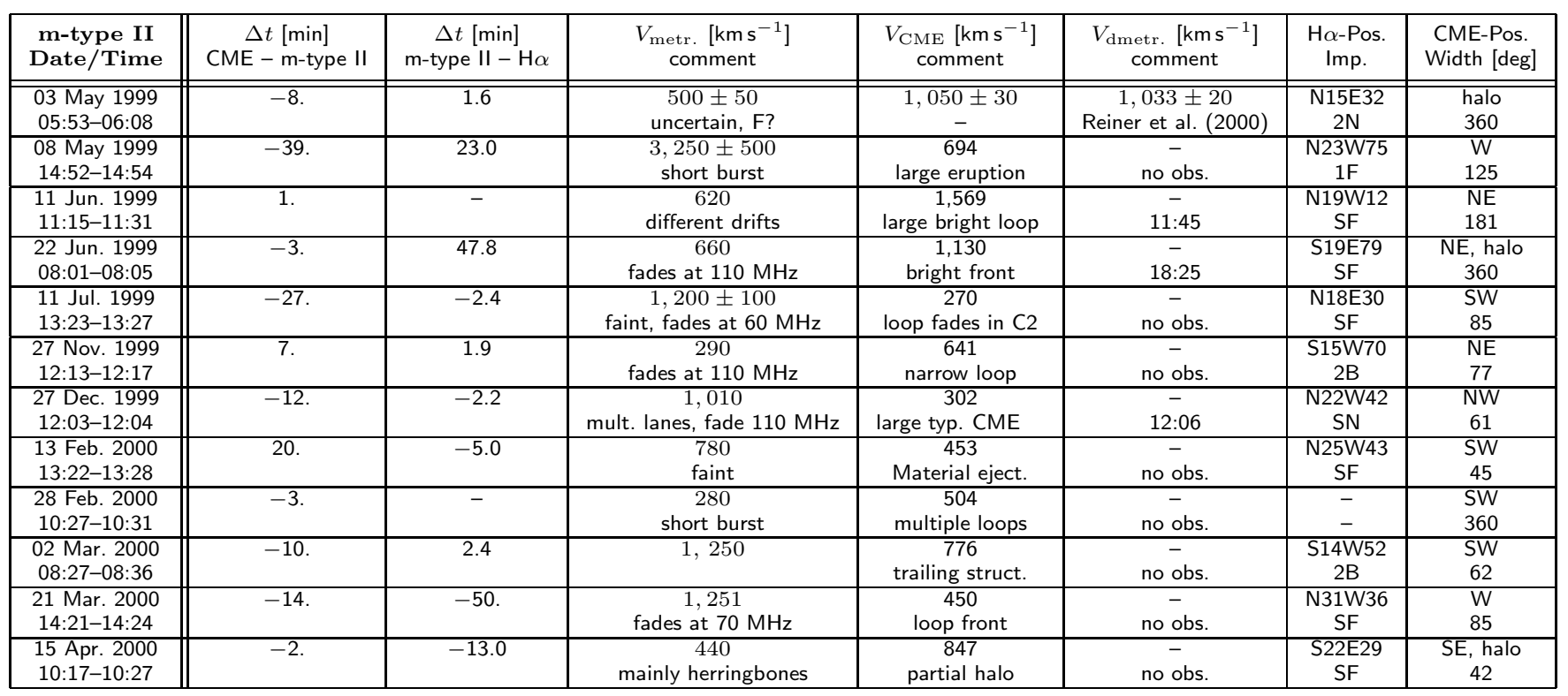


type II appearance leads the $\mathrm{H} \alpha$ maximum, respectively. Columns 4-6 contain the velocity of the CME, metric, and decametric type II source when available and a short comment on each event. The data for the decametric observations are not complete with respect to the velocity and the comment just states that Wind/WAVES observed decametric type IIs. Columns 7 and 8 contain the positions of the CMEs and $\mathrm{H} \alpha$ flares associated with the metric type IIs. Furthermore we indicated the importance of the flare and the observed width of the CME. This information will be used in the next section in order to discuss the visibility of CMEs with respect to the localization on the solar disc.

Looking at Table 1 and the corresponding data in Klassen et al. (2000) it should be realized that we distinguished two groups of events with respect to the velocity of the metric type IIs and CMEs from Cols. 4 and 5 . The reason for this is the fact that the velocity difference for the second group is hard to explain from the error/uncertainties discussed in the previous section. The use of different density models for the type IIs gives a maximum factor of 1.4, projection effects might add another factor of 1.4 (assuming a oblique propagation of $45 \mathrm{deg}$ ), and acceleration/deceleration of the CME might add a $100-200 \mathrm{~km} \mathrm{~s}^{-1}$ velocity difference. Thus we conclude that a factor of 2 should be the biggest systematic error for the metric type II velocity and that most events from the lower part of Table 1 differ by a factor of 2 or more. A similar argumentation was used by Reiner et al. (2000) for the event on 3 May, 1999 (see Table 1).

With respect to the decametric type IIs our study uses data either from the web or from publications. Without the data gaps of Wind/WAVES, we could compare the data from 61 metric type II and found in total $17(28 \%)$ decametric type IIs more or less temporally closely related to the metric type IIs. Furthermore we compared the metric - decametric type II association for the different classes of metric type IIs. Taking a temporal distance of 1 hour or more between CME and metric type II as criterion for class 1 this group consists of 19 metric type IIs and is associated with 5 decametric type IIs (26\%). The second group where the velocity between CME and metric type II differed by less than a factor of 2 is comprised of 19 events and is associated with 5 decametric type IIs (26\%). The last group associates 18 metric with 7 decametric type IIs (39\%).

\section{Discussion}

The data analysis of the previous section associated three classes of metric type IIs with CMEs. First, there is a group of metric type IIs with no clearcut observational relation to CMEs, either in white-light or in the decametric radio range. Secondly, there are events with an almost perfectly fitting kinematical behaviour, and thirdly there is a group of events with a close temporal association whereas the velocity correspondence is very poor. We drew - deduced from the histograms of Fig. 3 and an error estimation for the velocities - the dividing line between the different groups as follows: class 1: absolute time difference between CME and type II larger than 1 hour (19 events); class 2: absolute time difference smaller than 1 hour and velocity difference smaller than a factor of 2 (19 events); class 3: absolute time difference smaller than 1 hour and velocity difference larger than a factor of 2 (18 events).

Before we discuss these classes in greater detail it should be emphasized that both the dividing criteria and the assignment of the metric type II to the different classes are not incontrovertible. Some of these uncertainties could be removed by detailed case studies for each individual event. Thus it is very likely that the bad velocity correspondence for some events from Table 1 could be removed if we take for instance projection effects into account. Thus it might also be likely that bursts from class 2 might change to class 3 . But we do not expect that these changes lessen the evidence for the existence of different classes, although the number of events in each class might change slightly.

The first group comprises the metric type IIs with a time distance between metric type II and CME larger than 1 hour. Therefore it seems reasonable to consider a scenario where something is happening in the lower corona - a rising shock wave reaching up to $1 R_{\odot}$ above the photosphere - and we see almost nothing in LASCO C2 covering a heliocentric distance from $2.0-6 R_{\odot}$. Typical associates in LASCO are faint ejections/motions/changes as part of a CME being in progress. Furthermore it is well known that CMEs are best observed near the solar limb. On the other hand if there should be no direct CME - metric type II relation for this group of events, i.e., if we adopt the blast wave scenario, there should be a close correlation between flare and metric type II. In order to weigh up the alternative between almost invisible CMEs near the center of the solar disc and flare-related blast waves we looked at both the time difference between metric type II and $\mathrm{H} \alpha$ flare, and at the position of the flare.

Figure 4 shows the histograms of the aforementioned time difference for all three classes of metric type IIs. Here it is remarkable that the coincidence between flare and type II is best for class 1 , i.e., the type II with no obvious relation to CMEs. The upper panel shows only one coherent group of events while the other two classes are comprised of events with both a close temporal association and events that do not fit. Here it must be noticed that the outer bins around \pm 15 min are artificial blocks containing all events with a larger time difference than $15 \mathrm{~min}$ and that two metric type IIs from both class 2 and 3 could not be analysed with respect to a corresponding flare (cf. Table 1). The mean time difference plus standard deviation for the three classes is: $\left\langle\Delta t_{1}\right\rangle=2.9 \pm 4 \mathrm{~min}$, $\left\langle\Delta t_{2}\right\rangle=-3.4 \pm 10 \mathrm{~min}$, and $\left\langle\Delta t_{3}\right\rangle=1.0 \pm 9 \mathrm{~min}$. Thus, the small standard deviation for class 1 suggests that the type IIs in this class are most likely flare-related while the other two groups seem to be not directly flarerelated. 

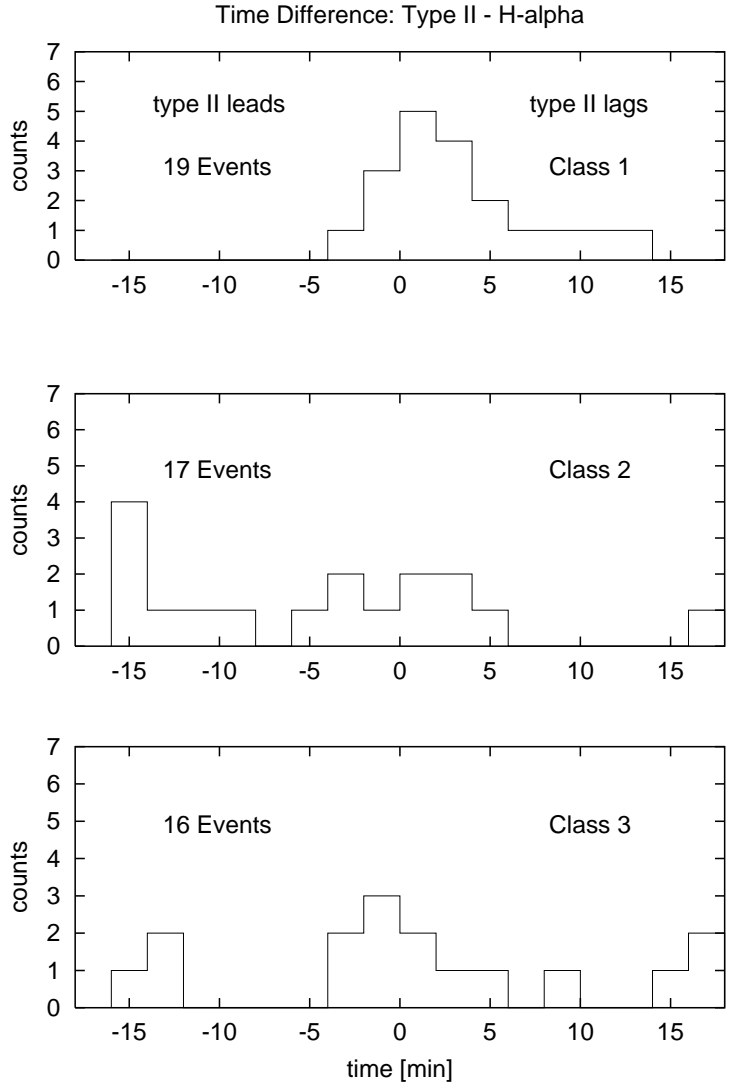

Fig. 4. Histogram of the time difference between the observed maximum of $\mathrm{H} \alpha$ flare and the onset of the metric type II. The three panels show the behaviour for the blast wave (class 1), the bow shock (class 2), and the internal part/flank (class 3) scenario.

Thus we conclude that the type IIs of the first class are the radio signatures of blast wave shocks during the flare phase only slightly perturbing the coronal regions observed in $\mathrm{C} 2$. These shocks are rising in the lower corona and dissipate somewhere in the $\mathrm{C} 2$ field of view. But one must be aware of the following caveat. Cliver et al. (1999) argue that the detection of faint CMEs is easier at the limb of the solar disc. Thus, if we make a simple classification between flares occurring in the center (within $\pm 30 \mathrm{deg}$ ) or limb-region $(\geq \pm 30 \mathrm{deg})$ of the solar disc, we find a ratio of 13:6 for class 1 and 9:10 for class 2. Furthermore it must be stated that all flares for the 12 events from the outer bins in Fig. 3 are occurring in the central part of the solar disc. That means that most of the flares associated with the type II of class 1 are observed close to the center of the solar disc, and we must expect that the LASCO observations for these events are less sensitive. One should analyse the corresponding events carefully. On the other hand it is the close temporal correlation with the $\mathrm{H} \alpha$ flare displayed in the upper panel of Fig. 4 which prompted us to adopt the flare-related blast wave scenario.

The data for the metric type IIs observed in 1999/2000 with a time distance to the CMEs smaller than 1 hour are displayed in Table 1. Our analysis showed that these transients are not homogeneous with respect to the driver velocities. Thus we divided the events into two subclasses as a dividing line we took a velocity difference larger than a factor of 2 which is hard to explain by systematic errors. But the first thing one should realize - either looking at Fig. 3 or at Table 1 - is that most of the CMEs precede the metric type II driver since the average time difference is negative $(\langle\Delta t\rangle=-8 \mathrm{~min})$. This means either that the source of the metric type II is following the CME leading edge or - if we adopt the bow shock scenario with the type II in front of the leading edge - that we are confronted with a small systematic error. One possible error could lie in acceleration/deceleration effects as second order corrections to the linear fits used for our analysis. Looking at the CME list at http://cdaw.gsfc.nasa.gov/CME_list it can be seen that most of the observed CMEs are decelerating and this would reduce the negative time difference. Since these effects as well as projection effects are not included in our study (for recent studies connecting CMEs and EIT observations see e.g. Thompson et al. 2000) we want to focus on the different density model, i.e. the enhancement factors as described in Sect. 2. These factors lead to a possible error of roughly $20 \%$ for the height of the $70 \mathrm{MHz}$ plasma level. This means that this plasma level might not correspond to a heliocentric distance of 1.5 but $1.2 R_{\odot}$. Therefore the CME height-time plots have to be extrapolated to a deeper layer of the corona. Assuming a CME speed of $500 \mathrm{~km} \mathrm{~s}^{-1}$ it takes 7 min to travel an additional distance of $0.3 R_{\odot}$ which might explain the negative time shift of 8 min.

Taking this consideration into account it seems possible to maintain the bow shock scenario if we change the density model from a fourfold to a onefold Newkirk model but we have to keep in mind that also the velocity of the type II would change (a decrease of about 20\%). In any case this change would not explain the factor 2 of the third group. In this respect it is worth mentioning that the average time shift for the two groups of Table 1 is different. We found time shifts of $\langle\Delta t\rangle=-5 \pm 10$ and $<\Delta t>=-10 \pm 13$ min for classes 2 and 3 , respectively. This means that the third class of metric type II may lie in events where the radio emission is generated behind the CME leading edge.

Thus we end up with some good evidence for three different scenarios in which metric type II bursts are involved. They might be called the blast wave, the bow shock, and the internal part or flank scenario. These results can be corroborated by further theoretical and observational results. From our observations we found that the first two classes (the blast waves and bow shocks) show $26 \%$ simultaneous metric and decametric type II observations each, and the third class (shocks at internal parts or flanks) 39\%. Especially, for the bow shocks we found a subgroup of 5 events where we also observed corresponding decametric type IIs. This means that the "perfect text-book examples" discussed in e.g. Kaiser et al. (1998), Mann et al. (1999), are indeed rare cases, less than $10 \%$ in our analysis. On the other hand it is striking that for the class of events when we commonly observe both 
metric and decametric type IIs - in the internal part or flank driven type IIs - the type IIs do not fit, a fact that is often claimed in the literature (e.g., Reiner et al. 2000, 2001a). In this case we are indeed looking at two different type II sources moving with different velocities.

This scenario is also observable in white-light where we are confronted with CMEs as spatially and temporally extended structures. Thus the CMEs analysed by Hundhausen et al. (1994) showed that different CMEfeatures, e.g., the outer loops, move with different velocities. But there are also theoretical arguments revealing different coronal shock scenarios. Thus the MHD simulations of Vršnak \& Lulić (2000a, 2000b) show that there are three scenarios of shock formation and propagation, namely blast waves, piston-driven shock, and bow shocks. Thus it might be possible that we observe internal parts of the CME - what these authors call the piston - in the metric radio range and we observe the leading edge in white-light. In other words, we are observing two different parts of the same spatially structured transient, each in different part of the electromagnetic spectrum.

Finally, our data suggest that the relative occurrence of different kinds of metric type IIs varies over the solar cycle, as can be deduced from Fig. 3. The reason for this behaviour might be connected to the conditions for type II radio emission and variations of CME properties during the solar cycle. A simple explanation would make use of critical shock speeds to generate radio waves. Thus Benz \& Thejappa (1988) or Mann et al. (1995) argue that only so-called supercritical shocks are type II generating. In addition it seems very likely that the shock geometry is also a key player with respect to the acceleration of electrons that are the starting point for the radio wave generation. Looking at in-situ measurements of interplanetary shocks Claßen et al. (1998) found the highest electron fluxes at supercritical quasi-perpendicular shocks, i.e., at shocks moving with an oblique angle of roughly $60^{\circ}$ to the magnetic field. Thus it is very likely that only shocks with a sufficiently high shock speed and an appropriate shock geometry are able to generate type II radio emission. Therefore it is obvious to look for CME variations over the solar cycle. Thus, Hundhausen et al. (1984) reported on CME observations just at low latitudes during solar minimum while they observed CME at all latitudes during solar maximum. In addition to that Hundhausen et al. (1994) discussed annual changes of the CME speed over the solar cycle and that the latitudinal behaviour of CME during the solar cycle agrees with the latitude of coronal helmet streamers (Hundhausen 1993). In order to avoid a misunderstanding it should be mentioned that there is no evidence for a general solar cycle modulation of the CME speed but rather periods within the solar cycle with significantly different mean velocities (St. Cyr, private communication). This might be the case for the events in 1997 as observed by Klassen et al. (2000).

For our own data sample we found the following results. The mean speed of the CMEs analysed in our paper was $490 \pm 200 \mathrm{~km} \mathrm{~s}^{-1}$ and $690 \pm 250 \mathrm{~km} \mathrm{~s}^{-1}$ for the events in 1997 and 1999/2000, respectively. In contrast the velocities of the corresponding coronal type IIs were $740 \pm 220 \mathrm{~km} \mathrm{~s}^{-1}$ and $770 \pm 350 \mathrm{kms}^{-1}$ for 1997 and $1999 / 2000$, respectively. Here it should be recalled that Klassen et al. (2000) used the $1 \times$ Newkirk model. This means being consistent with the density model of the present paper that we have to increase the mean velocity of the events observed in 1997 by a factor of 1.4, i.e., we obtain a mean type II source velocity of $1000 \pm 300 \mathrm{~km} \mathrm{~s}^{-1}$ in 1997. Looking at these velocities it should be realized that the mean CME speed increases from 1997 to 1999/2000 while the mean speed of the metric type II sources decreases. This could mean that the slow CMEs in 1997 are almost invisible in the radio range because they are no longer supercritical and we are merely observing metric type IIs related to fast blast wave shocks. Thus it seems very likely that most of the metric type IIs in 1997 are related to flares with fast blast wave shocks - with no direct relation to the $\mathrm{CME}$ leading edge motion - while the metric type IIs in the $1999 / 2000$ events are generated by both blast wave and CME-driven shocks.

\section{Summary and conclusions}

Revising the data archives with respect to the significance of coronal type II bursts we showed that there are at least two different scenarios in which these radio bursts are involved. We analysed a group of 63 coronal type IIs and found that roughly $30 \%$ of these events show no obvious relation to white-light CME observations. But on the other hand we argued that another $30 \%$ agree very well with respect to velocity and extrapolated onset. Thus these two groups of events almost perfectly fit the blast wave and piston-driven shock paradigms. Furthermore our analysis showed (cf. Fig. 3) that the relative occurrence of the two groups might be solar cycle dependent. For the solar minimum events from 1997 we mainly observed type II bursts associated with blast waves and during solar maximum we observe a mixture of blast wave generated type IIs and CME-associated type IIs. Thus it would be possible to explain some diverging results discussed in the literature (cf. Aurass 1997). In order to corroborate the solar cycle dependence and to improve the statistics an extended analysis is necessary. This should be possible due to the long-lasting observations of ground-based radio spectrometers and space-borne coronagraphs.

Furthermore, we found a CME related group of 18 coronal type IIs (29\%) which give rise to a more complex point of view. We argued that these observations might be explained by a type II generation at internal parts or flanks of the corresponding CMEs. This would split up the simple piston-driven paradigm into a pure bow shock and a multiple shock scenario which might also be related to blast waves as triggering events. Therefore a detailed analysis by interferometric radio observations is necessary.

Finally we made a compilation of metric and decametric type IIs. We found that the number of consecutive 
coronal and interplanetary type II burst observations in the different event categories increases from blast waves ( 5 from in total 19 events) and bow shocks ( 5 from in total 19 events) to piston-driven shocks ( 7 from in total 18 events). This result might explain why perfectly fitting metric and decametric type IIs are very rarely reported - according to our estimation less than 10\% - and why most of the decametric type IIs trace different parts of the CMEs than the metric type IIs. Thus their heighttime plots are not compatible.

Acknowledgements. We thank O. C. St. Cyr and S. P. Plunkett for analysing the CME data and for the lively and stimulating discussion. We used the CME data from http://cdaw.gsfc.nasa.gov/CME_list. This CME catalog is generated and maintained by the Center for Solar Physics and Space Weather, The Catholic University of America in cooperation with the Naval Research Laboratory and NASA. $\mathrm{SOHO}$ is a project of international cooperation between ESA and NASA. The Wind/WAVES data were taken from the Wind/WAVES webpage (http://lep694.gsfc.nasa.gov/ waves/waves.html). We thank M. J. Reiner for the discussion about our results. The work of H. T. Claßen is financially supported by DLR (grant No. 9012).

\section{References}

Aurass, H. 1997, Coronal Mass Ejections and type II Radio Bursts, in Coronal Physics from Radio and Space Observations, ed. G. Trottet (Springer, Berlin, New York, Lecture Notes in Physics), 483, 135

Aurass, H., Hofmann, A., \& Urbarz, H.-W. 1998, A\&A, 334, 289

Bastian, T. S., Pick, M., Kerdraon, A., Maia, D., \& Vourlidas, A. 2001, ApJ, 558, L65

Benz, A. O., \& Thejappa, G. 1988, A\&A, 202, 267

Bougeret, J.-L. 1985, Observations of Shock Formation and Evolution in the Solar Atmosphere, in Collisionless Shocks in the Heliosphere: Reviews of Current Research, ed. B. T. Tsurutani, \& R. G. Stone (AGU Geophys. Monograph 34, Washington DC), 13

Bougeret, J.-L., Kaiser, M. L., Kellogg, P. J., et al. 1995, Space Sci. Rev., 71, 231

Brueckner, G. E., Howard, R. A., Koomen, M. J., et al. 1995, Sol. Phys., 162, 357

Cairns, I. H., \& Robinson, R. D. 1987, Sol. Phys., 111, 365

Claßen, H. T., Mann, G., \& Keppler, E. 1998, A\&A, 335, 1101

Cliver, E. W., Webb, D. F., \& Howard, R. A. 1999, Sol. Phys., 104, 4743

Dulk, G. A., Leblanc, Y., Bastian, T. S., \& Bougeret, J.-L. 2000, J. Geophys. Res., 105, 27, 343

Ginzburg, V. L., \& Zheleznyakov, V. V. 1958, Astron. Zh., 35, 694; transl. Sov. Astron. A. J., 2, 653

Gopalswamy, N., Kaiser, M. L., Lepping, R. P., et al. 1998, J. Geophys. Res., 103, 307
Gopalswamy, N. 2000, Type II Solar Radio Bursts, in Radioastronomy at Long Wavelengths (AGU Geophys. Monograph 119, Washington, DC), 123

Hildner, E. 1977, Mass ejections from the solar corona into interplanetary space, in Studies of Travelling Interplanetary Phenomena, ed. M. A. Shea, D. F. Smart, \& S. T. Wu (Reidel, Dordrecht), 3

Hundhausen, A. J., Sawyer, C. B., House, L. L., Illing, R. M. F., \& Wagner, W. J. 1984, J. Geophys. Res., 89, 2639

Hundhausen, A. J. 1993, J. Geophys. Res., 98, 13177

Hundhausen, A. J., Burkepile, J. T., \& St. Cyr, O. C. 1994, J. Geophys. Res., 99, 6543

Kaiser, M. L., Reiner, M. J., Gopalswamy, N., et al. 1998, Geophys. Res. Lett., 25, 2501

Khan, J. I., \& Aurass, H. 2002, A\&A, 383, 1018

Klassen, A., Aurass, H., Klein, K.-L., Hofmann, A., \& Mann, G. 1999, A\&A, 343, 287

Klassen, A., Aurass, H., Mann, G., \& Thompson, B. J. 2000, A\&AS, 141, 357

Klein, K.-L., Klassen, A., Aurass, H., \& LASCO-Consortium 1997, ESA-SP404, 461

Mann, G., Aurass, H., Voigt, W., \& Paschke, J. 1992, ESA SP-348, 129

Mann, G., Claßen, H. T., \& Aurass, H. 1995, A\&A, 295, 775

Mann, G., Klassen, A., Claßen, H. T., et al. 1996, A\&AS, 119, 489

Mann, G., Aurass, H., Jansen, F., et al. 1999, AIP Preprint Series, AIP 99-12

Nelson, G. S., \& Melrose, D. B. 1985, Type II Bursts, in Solar Radiophysics, ed. D. J. McLean, \& N. R. Labrum (Cambridge Univ. Press, Cambridge), 333

Newkirk, G. Jr. 1961, ApJ, 133, 983

Newkirk, G. Jr. 1967, ARA\&A, 5, 213

Reiner, M. J., Fainberg, J., Kaiser, M. L., \& Stone, R. G. 1998, J. Geophys. Res., 103, 1923

Reiner, M. J., Kaiser, M. L., \& Bougeret, J.-L. 2000, ApJ, 529, L53

Reiner, M. J., Kaiser, M. L., Gopalswamy, N., et al. 2001a, J. Geophys. Res., 106, 25279

Reiner, M. J., Kaiser, M. L., \& Bougeret, J.-L. 2001b, J. Geophys. Res., 106, 29989

Robinson, R. D., \& Stewart, R. T. 1985, Sol. Phys., 97, 145

Saito, K. A. 1970, Ann. Tokyo Aston. Obs., Ser. 2, 12, 53

Saito, K. A., Poland, A. I., \& Munro, R. H. 1977, Sol. Phys., 55,121

Sheeley, N. R., Stewart, R. T., Robinson, R. D., et al. 1984, ApJ, 279, 839

St. Cyr, O. C., et al. 2000, J. Geophys. Res., 105(18), 169

Thompson, B. J., Cliver, E. W., Nitta, N., Delannée, C., \& Delaboudinière 2000, Geophys. Res. Lett., 27(1), 413

Uchida, Y. 1960, PASJ, 12, 376

Vršnak, B., \& Lulić, S. 2000a, Sol. Phys., 196, 157

Vršnak, B., \& Lulić, S. 2000b, Sol. Phys., 196, 181

Warmuth, A., Vršnak, B., Aurass, H., \& Hanslmeier, A. 2001, ApJ, 560, L105

Wild, J. P. 1962, J. Phys. Soc. Jpn, 17 (Suppl. A-11), 249 